\section{A-head of the rest}

Minimising the amount of dental equipment in a surgery makes sense financially as well as logistically. The VistaCam iX HD, from Durr Dental, does just that, since it has an interchangeable head so that you can use it for intraoral, extraoral and macro images (using the 'Cam' head) as well as to detect carious lesions and display plaque (courtesy of the 'Proof' head) in addition to early detection of proximal caries (using the 'Proxi' head). The concept is simple, the technology unrivalled, the possibilities endless.

An intraoral camera can provide both dentists and their patients with a revealing window into the realm of dental disease, conferring greater transparency and more lucid communication. The Cam head of the VistaCam iX HD delivers crystal clear high definition images, thanks to the variable autofocus, regardless of whether you require intraoral, extraoral or macro images. Videos can also be recorded in the same HD resolution. Twin LEDs illuminate the oral cavity. The implications for patient communication and treatment are clear: seeing really is believing!

The Proof head detects any carious lesions, rates them on a colour scale and assigns them a numerical value ranging from green $(0-1.0)$ for healthy enamel to yellow $(>2.5)$ for deep dentine caries. This intuitive software spots early lesions and presents the evidence clearly for you to show patients. It can also be used to monitor the progression of disease, should a patient choose not to

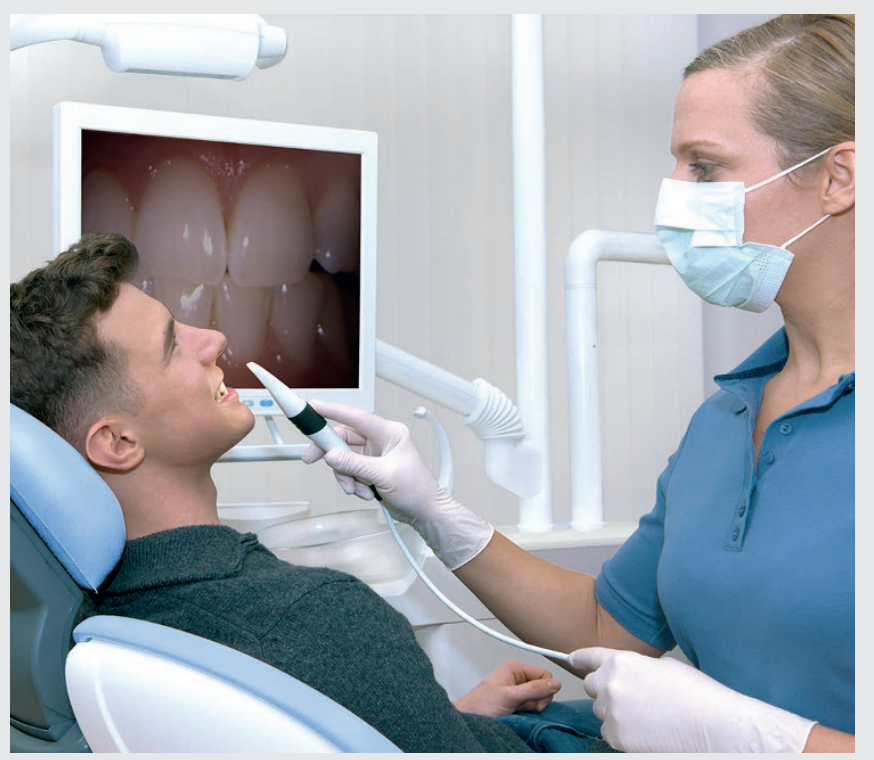

act in the early stages. It can also be used by hygienists for pinpointing plaque: no more messy disclosing tablets!

The VistaCam iX HD offers unrivalled functionality in a single device with multiple applications, perfectly complementing daily practice with an indispensable tool.

\title{
World's first natural dry mouth rinse
}

Dr Harold Katz and his No 1 breath care brand The Breath Company in the USA has launched a revolutionary and world-first dry mouth rinse to contain natural flower derivatives and moisturisers.

The Breath Company Dry Mouth Rinse is clinically proven to be naturally effective at controlling and soothing dry mouth conditions without drying alcohol or staining compounds. In clinical tests $95.4 \%$ of users said it helped reduce dryness and soothed their mouth, $97.1 \%$ strongly agreed it was very refreshing, $96.2 \%$ said it moisturised their mouth and $75 \%$ of users said they would switch

from their previous mouthwash.

Combining natural moisturisers, salivary enzymes and a salivary stimulant, The Breath Company Dry Mouth Rinse works to reverse the signs of xerostomia and help reduce the frequency of recurring dry mouth symptoms by stimulating, lubricating, moisturising, soothing and refreshing the mouth for hours.

Designed to work instantly and to keep working for hours to keep the mouth moist using its specialist moisturising technology, The Breath Company Dry Mouth Rinse works on all types of dry mouth conditions and contains no harsh ingredients such as alcohol, detergents, artificial flavours, colours, or animal products or gluten.

The Breath Company Dry Mouth Rinse is available in Boots stores nationwide and online at www.boots.com, priced at $£ 14.00$.

\section{New website for remineralising toothpaste brand}

BioMin Technologies, the company behind the toothpaste which reduces tooth sensitivity, helps replace lost mineral from tooth surfaces and protects against decay, has launched its new website: www.biomin.co.uk.

The new website will enable more effective engagement with consumers, dentists, the dental industry and scientists. It will also provide greater clarity and ease of access to content.

The new website features:

- Deeper and broader content - around the company, its products and the science and technology behind the products. This includes text, diagrammatic and video explanations, new scientific data and references to scientific and clinical studies

- Easy navigation

- A fully responsive design - mobile and tablet friendly

- A new and effective search function

- Contemporary design and layout - modern, intuitive and clean layout with a clear and integrated user experience and improved visual identity.

The new website will help BioMin Technologies explain how and why its development of mineral-releasing glass additives for oral and dental care products will improve the oral health of the general public.

BioMin Technologies is preparing to launch its second product, the BioMin C Fluoride free toothpaste, early this autumn.

www.biomin.co.uk 